\title{
On Star Formation in TDC
}

\author{
A. S. Hojaev and S. N. Nuritdinov \\ Astronomy Dept., National University of Uzbekistan, Tashkent, 700174, Uzbekistan \\ email: ash@astrin.uzsci.net
}

\begin{abstract}
The value of different forms of energy contained in TDC region as well as the structure and physical characteristics of star formation in the Taurus Dark Clouds are analyzed.
\end{abstract}

Keywords. (Galaxy:) open clusters and associations: general; stars: formation

\section{Introduction}

Taurus dark cloud region (TDC) is one of the nearest $(\mathrm{D} \approx 135 \mathrm{pc})$ star formation nests in the Galaxy with the averaged total mass of the cloud material in the complex estimated as $4500 M_{S}$ (Jones \& Herbig 1979). 4 T-associations and no OB-association are in the region of TDC. The flare stars, IR sources, HHOs, WTTS X-ray sources as well as many PMS-star candidates and brawn dwarfs have been found in TDC in the last years (see, e.g., Scelsi et al. 2007). Pleiades located practically at the same distance from the Sun is closely adjoin to TDC and separates from TDC for about $10 \mathrm{pc}$ only. Eggen (1995) ascribes Pleiades cluster, some TTS of its vicinity and Persei cluster to the united Pleiades supercluster.

\section{Calculations}

The careful calculation of the total mass of already formed stars based on recent observational data gives the estimation of stellar content mass $150-200 M_{S}$. This leads to the SFE of about 3-4\%. This value is $\ll$ SFE in Orion and Perseus complexes $(\sim 20 \%$, Vrba 1977). Clearly the star formation process in the TDC closely correlates with physical conditions in the region as well as with the total energy balance in the region according to the Jeans instability criterion. The calculation of the complex total energy components is necessary also to estimate the extent of complex instability in time in accordance with a virial theorem, and to define the current physical conditions. Thus we computed the approximate value of the different forms of energy for considered region of TDC . The potential energy of the Galaxy's gravitational field influence upon the TDC

$$
\Omega_{G}=-M_{T D C} U=-\frac{M_{T D C} U_{0}}{1+\chi D_{T D C}^{2}+b z_{T D C}^{2}},
$$

where $U$ is the potential of gravitation energy of the Galaxy; $D_{T D C}$ is the distance of the region from the Galaxy center; $z_{T D C}$ - the distance of the region from the Galaxy disk.

Taking into account the proximity of the region to the Galaxy disk $\left(z_{T D C}<<D_{T D C}\right)$, in first approximation one can neglect of third term in the denominator . The $U_{0}$ and $\chi$ values could be easily computed through the Oort constants. The distance of the TDC from the Galaxy center is calculated as $D_{T D C}=10.124 \mathrm{kpc}$. Taking into account the mean combined mass of the stellar and pre-stellar matter (molecular, gas-dust), belonging 
to the TDC as $4700 M_{S}$ we find $\Omega_{G} \approx-4.8 \cdot 10^{51} \mathrm{erg}$. The proper potential gravitational energy of the TDC molecular and gas-dust clouds is derived as

$$
\Omega_{T D C}=-\frac{3}{5} \frac{G M_{T D C}}{R_{T D C}}
$$

If one assume the region's radius as half-sum of its semi- axes, than taking into account the distance between the Sun and the TDC as large as $135 \mathrm{pc}, R_{T D C}=7 \mathrm{pc}, \Omega \approx$ $-1.5 \cdot 10^{47} \mathrm{erg}$.

To compute the magnetic field energy in the TDC let us consider the spherically symmetric approximation in the same way as in case of energy of the proper gravitational field

$$
M=\frac{1}{6} H^{2} R_{T D C}^{3},
$$

where $H$ is the strength of magnetic field in TDC. The value $H<50$ Gauss, given in the paper by Crutcher et al. (1975) is an upper limit estimation of magnetic field strength in the TDC, found based on observations in the radio range of Zeeman's splitting of the hydroxyl line. Therefore the value of magnetic energy $\leqslant 4.2 \cdot 10^{48} \mathrm{erg}$ represents the maximally possible value, i.e., is also an upper limit estimation of magnetic field energy.

The complex of clouds in TDC was found to be in rotation (Ho et al. 1977) and its kinetic energy of rotation

$$
T_{m r}=\frac{1}{2} I_{T D C} \omega^{2}=\frac{1}{2} M_{T D C} R_{T D C}^{2} \omega^{2},
$$

where $I_{T D C}$ is the momentum of inertia of the complex; $\omega$ - angular velocity of its rotation. When $\omega=6.4 \cdot 10^{-14} \mathrm{radian} / \mathrm{s}, T_{m r} \approx 3.4 \cdot 10^{48} \mathrm{erg}$. The kinetic energy of turbulent and convective gas motions in the TDC

$$
T_{m t}=\frac{1}{2} M_{T D C} v^{2}
$$

If accept as velocity of these motions the value $v_{D}=0.27 \mathrm{~km} / \mathrm{s}$ (Ho et al. 1977$)$ - the value of Doppler's widening of the ammonia $\mathrm{NH}_{3}$ molecules radio-line, which is significantly narrower than other molecular lines because of more compactness of ammonia clots, and therefore the impact of large scale rotation of the TDC should be slight, than $T_{m t} \approx 3.3 \cdot 10^{45} \mathrm{erg}$.

And, at last, the kinetic energy of thermal molecular motion in the clouds complex upon its considering as spherically symmetric ideal gas with the radius $R_{T D C}$, mass $M_{T D C}$, pressure $\mathrm{P}$ and temperature $\mathrm{T}$ :

$$
T_{k}=\frac{3}{2} \frac{M_{T D C} k T}{\mu m^{H}}=4 \pi P R_{T D C}^{3}+3(\gamma-1) U
$$

where $\mu$ is the mean molecular mass of the gas; $m_{H}$ is the hydrogen atom's mass; $k$ is the Boltzmann's constant; is the adiabatic (isentropic) exponent; $U$ is the internal (intrinsic) energy, conditioned by thermal molecular motion. Apparently, this energy could be estimated by the following formula with universal (or molar) gas constant $R$

$$
T_{k}=3 R M_{T D C} T
$$

When average temperature of TDC $=12$ (Ho et al. 1977 ), $T k \approx 2.7 \cdot 10^{46} \mathrm{erg}$.

According to a virial theorem the system is in equilibrium if

$$
2 T+\Omega=0,
$$


where $T$ and $\Omega$ are the total kinetic and the total potential energies, respectively Consequently the quantity

$$
\alpha=\frac{2 T}{\Omega}
$$

is characterizing the system divergence from the equilibrium state. The virial parameter $\alpha<<1$, i.e., the region considered is manifestly non-stationary and process of star formation in this region continues. As regards the total energy of the TDC, the calculations indicate its obvious negativity $\left(-10^{51} \mathrm{erg}\right)$.

\section{References}

Jones, B. F. \& Herbig, G. H. 1979, AJ, 84, 1872

Scelsi, L., Maggio, A., Micela, G., Pillitteri, I., Stelzer, B., Briggs, K., Guedel, M., Grosso, N., Audard, M., \& Palla, F. 2007, A\&A, 468, 405

Eggen, O. J. 1995, AJ, 110, 1749

Vrba, F. J. 1977, AJ, 82, 198

Crutcher, R. M., Evans, N. J., Troland, T., \& Heiles, C. 1975, ApJ , 198, 91

Ho, P. T. P., Martin, R. N., Myers, P. C., \& Barrett, A. H. 1977, ApJ (Letters), 215, L29 